\title{
Leakage radiation microscopy of surface plasmon polaritons
}

\author{
A. Drezet, A. Hohenau, D. Koller, A. Stepanov, H. Ditlbacher, \\ B. Steinberger, F. R. Aussenegg, A. Leitner, and J. R. Krenn \\ Institute of Physics, Karl-Franzens University Graz, \\ Universitätsplatz 5 A-8010 Graz, Austria
}

(Dated: October 29, 2018)

\begin{abstract}
We review the principle and methodology of leakage radiation microscopy (LRM) applied to surface plasmon polaritons (SPPs). Therefore we first analyse in detail the electromagnetic theory of leaky SPP waves. We show that LRM is a versatile optical far-field method allowing direct quantitative imaging and analysis of SPP propagation on thin metal films. We illustrate the LRM potentiality by analyzing the propagation of SPP waves interacting with several two dimensional plasmonic devices realized and studied in the recent years.

PACS numbers:
\end{abstract}




\section{INTRODUCTION}

In recent years intensive investigations of surface plasmon polaritons (SPPs) have been made in the promising context of nanophotonics. This research is actually motivated by the current trends for optical device miniaturisation and by the possibilities of merging aspects of nanophotonics with those of electronics. SPPs are electromagnetic waves bounded to dielectric-metal interface. As surface waves, SPPs are exponentially damped in the directions perpendicular to the interface [1]. Furthermore, SPPs could be used to transfer optical information in a two dimensional (2D) environment. This appealing property can be used for optical addressing of different 2D optical systems and nanostructures located at a dielectric/metal interface. Actually several 2D SPP devices including passive nanostructures including mirrors or beam splitter and active elements like molecules or quantum dots are currently under development and investigation. Developments such as these raise the prospect of a new branch of photonics using SPPs, for which the term "plasmonics" emerged [2 4]].

However, for experimental investigations of optical devices an important characteristic of SPP modes is that their spatial extent is governed and defined by the geometry of the nanoelements rather that by the optical wavelength [7]. This consequently opens possibilities for breaking the diffraction limit but requires instruments of observation adapted essentially to the subwalength regime and being capable of imaging the propagation of SPPs in their 2D environment. Usually the analysis of the subwalength regime implies necessarily near field optical (NFO) methods [5, 6] able to collect the evanescent (i. e., non radiative) components of the electromagnetic fields associated with SPPs. However, when the metal film on which the 2D optical elements are built is thin enough (i. e ., with a thickness below 80-100 $\mathrm{nm}$ ) and when the subtratum optical constant (usually glass) is higher than the one of the superstratum medium an other possibility for analyzing SPP propagation occurs. This possibility is based on the detection of coherent leaking of SPPs through the substratum.

Such a far-field optical method is called leakage radiation microscopy (LRM) [8-10] and allows indeed a direct quantitative imaging and analysis of SPP propagation on thin metal films.

The aim of this article is to present a short overview of recent progress in the field of SPP 
imaging using LRM. In a first part of this work we will describe the theoretical principles underlying LRM. In the second part we will discuss modern leakage radiation methods and illustrate the LRM potentialities by analyzing few experiments with SPP waves interacting with $2 \mathrm{D}$ plasmonic devices.

\section{LEAKAGE RADIATION AND SURFACE PLASMON POLARITONS}

In order to describe the theoretical mechanisms explaining leakage radiation it will be sufficient for the present purpose to limit our analysis to the case of a metal film of complex permittivity $\epsilon_{1}(\omega)=\epsilon_{1}^{\prime}+i \epsilon_{1}^{\prime \prime}(\omega=2 \pi c / \lambda$ is the pulsation) sandwiched between two dielectric media of permittivity $\epsilon_{0}$ (substrate) and $\epsilon_{2}<\epsilon_{0}$ (superstratum). This system is theoretically simple and to a good extent experimentally accessible [1, 11]. In the limiting case where the film thickness $D$ is much bigger than the SPP penetration length in the metal (i. e., $D \gtrsim 70$ $\mathrm{nm}$ for gold or silver in the visible domain) one can treat the problem as two uncoupled single interfaces. We will consider as an example the interface $0 / 1$ (the media 0 and 1 are located in the domain $z \geq 0$ and $z \leq 0$ respectively ). Such an interface will be identified in the following with the plane $z=$ const. in cartesian coordinates. An elementary harmonic SPP wave is actually a TM electromagnetic mode characterized by its pulsation $\omega$ and its magnetic field $\mathbf{H}=\left[0, H_{y}, 0\right]$ where the y component can be written

$$
\begin{gathered}
H_{0}=\alpha e^{i k_{x} x} e^{i k_{z 0} z} e^{-i \omega t} \text { in the medium } 0 \\
H_{1}=e^{i k_{x} x} e^{i k_{z 1} z} e^{-i \omega t} \text { in the medium } 1
\end{gathered}
$$

and where $k_{x}=k_{x}^{\prime}+i k_{x}^{\prime \prime}$ is the (complex valued) wavevector of the SPP propagating in the $\mathrm{x}$ direction along the interface. $k_{z j} \equiv k_{j}= \pm \sqrt{\left[(\omega / c)^{2} \epsilon_{i}-k_{x}^{2}\right]}$ are the wave vectors in the medium $\mathrm{j}=[0$ (dielectric), 1 (metal) $]$ along the direction $z$ normal to the interface. By applying boundary conditions to Maxwell's equations one deduces additionally $\alpha=1$ and

$$
\frac{k_{1}}{\epsilon_{1}}-\frac{k_{0}}{\epsilon_{0}}=0,
$$

which implies

$$
\begin{aligned}
& k_{x}= \pm(\omega / c) \sqrt{\frac{\epsilon_{0} \epsilon_{1}}{\epsilon_{0}+\epsilon_{1}}} \\
& k_{j}= \pm(\omega / c) \sqrt{\frac{\epsilon_{j}^{2}}{\epsilon_{0}+\epsilon_{1}}}
\end{aligned}
$$


for a SPP wave propagating along the $\mathrm{x}$ direction. The choice of the sign convention connecting the $\mathrm{z}$ and $\mathrm{x}$ components of the wave vector is a priori arbitrary and must be done only on a physical ground. Indeed, due to ohmic losses in the metal we expect an expo-

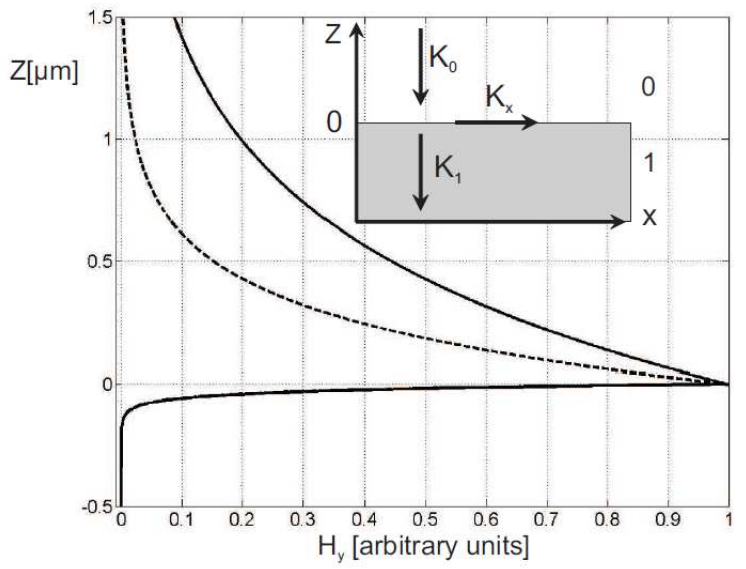

FIG. 1: Structure of the SPP magnetic field Real $\left[H_{y}\right]$ across an interface air/gold (thick line) and glass/gold (dashed line). The optical wavelength considered is $\lambda=800 \mathrm{~nm}$. The permittivity of glass is taken to be $\epsilon_{\text {glass }}=2.25$. The inset shows the conventions for the axes $\mathrm{x}$ and $\mathrm{z}$. The arrows indicate the direction of the real part of the wave vector normally and parallely to the interface.

nentially decaying SPP wave propagating along the interface. This condition implies the relation $k_{x}^{\prime} \cdot k_{x}^{\prime \prime} \geq 0$ [11]. This inequality is actually always fulfilled since from Eq. 3 one deduces

$$
k_{x}^{\prime} \cdot k_{x}^{\prime \prime}=\frac{1}{2}(\omega / c)^{2} \frac{\epsilon_{0}^{2} \epsilon_{1}^{\prime \prime}}{\left(\epsilon_{0}+\epsilon_{1}^{\prime}\right)^{2}+\left(\epsilon_{1}^{\prime \prime}\right)^{2}}>0
$$

which is indeed positive because $\epsilon_{1}^{\prime \prime}>0$. By writing $k_{z j}=k_{j}^{\prime}+i k_{j}^{\prime \prime}$ one additionally obtains the relation

$$
-k_{0}^{\prime} \cdot k_{0}^{\prime \prime}=(\omega / c)^{2} \epsilon_{1}^{\prime \prime} / 2-k_{1}^{\prime} \cdot k_{1}^{\prime \prime}=k_{x}^{\prime} \cdot k_{x}^{\prime \prime} \geq 0
$$

This relation fixes the sign conventions since the wave must also decay exponentially when going away from the interface in both media. More precisely one gets

$$
\begin{gathered}
k_{0}^{\prime} \cdot k_{0}^{\prime \prime} \leq 0, \\
k_{1}^{\prime} \cdot k_{1}^{\prime \prime}=\geq 0
\end{gathered}
$$

The product $k_{1}^{\prime} \cdot k_{1}^{\prime \prime}$ is positive if $\epsilon_{1}^{\prime} \geq-\left|\epsilon_{1}\right|^{2} /\left(2 \epsilon_{0}\right)$, a fact which is indeed true for silver and gold interfaces with air or glass in most of the visible optical domain. However small negative 
values of Eq. 8 occur for silver close to the interband region around $\lambda \sim 350 \mathrm{~nm}$. Additionally a higher value of $\epsilon_{0}$ will also change the sign in Eq. 8. Fig. 1 shows the behavior of the SPP magnetic field close to an interface gold/air and gold/glass at the optical wavelength $\lambda=800$ nm. At such a wavelength the conditions given by Eqs. 5-8 impose the solutions

$$
k_{x}= \pm(\omega / c) \sqrt{\frac{\epsilon_{0} \epsilon_{1}}{\epsilon_{0}+\epsilon_{1}}}, k_{j}=-(\omega / c) \sqrt{\frac{\epsilon_{j}^{2}}{\epsilon_{0}+\epsilon_{1}}} .
$$

The real parts of the $k_{z}$ components of the SPP wave vector are for both media oriented in the same direction corresponding to a wave propagating from the air side to the metal side (see inset in Fig. 1). Furthermore the waves are exponentially damped when going away from the interface in agreement with Eq. 7, 8 (see Fig. 1). Most important for us is that the Poynting vector [12] $\mathbf{S}=\operatorname{Real}\left[\mathbf{E} \times \mathbf{H}^{*}\right] / 2$ is defined in the medium $\mathrm{j}$ by

$$
\mathbf{S}_{j}=\frac{1}{2} c \operatorname{Real}\left[\frac{k_{x} \hat{\mathbf{x}}+k_{j} \hat{\mathbf{z}}}{\omega \epsilon_{j} / c}\right] e^{-2 k_{x}^{\prime \prime} x-2 k_{z}^{\prime \prime} z} .
$$

On the dielectric side the energy flow is as expected oriented in the direction of Real[k]. However it can be shown on the metal side and for wavelengths not too close from the spectral region associated with the interband transition of gold or silver that the energy flow in the $\mathrm{x}$ direction is oriented oppositely to the wave vector Real $\left[k_{x}\right]$ since Real $\left[k_{x} / \epsilon_{1}\right]=$ $\left(k_{x}^{\prime} \epsilon_{1}^{\prime}+k_{x}^{\prime \prime} \epsilon_{1}^{\prime \prime}\right) /\left|\epsilon_{1}\right|^{2}$ is dominated by $k_{x}^{\prime} \cdot \epsilon_{1}^{\prime}$ and since $\epsilon_{1}^{\prime}<0$. However the total energy flow in the x direction $S_{x}=\int_{0}^{+\infty} S_{x}^{(\text {air })} d z+\int_{-\infty}^{0} S_{x}^{(\text {metal })} d z$ is oriented along $k_{x}^{\prime}$. Additionally in the $\mathrm{z}$ direction $\operatorname{Real}\left[k_{1} / \epsilon_{1}\right]=\left(k_{1}^{\prime} \epsilon_{1}^{\prime}+k_{1}^{\prime \prime} \epsilon_{1}^{\prime \prime}\right) /\left|\epsilon_{1}\right|^{2}$ is parallel to $k_{1}^{\prime}$ since $k_{1}^{\prime \prime} \epsilon_{1}^{\prime \prime}$ dominate. This implies in particular that the energy associated with the SPP wave is absorbed by the metal during its propagation through the interface from the air side to the glass side. It should be observed that already in the case of the ideal plasma model neglecting losses the wave vector $k_{x}=k_{x}^{\prime}$ is antiparallel to $S_{x}$ in the metal since there is no imaginary part and since $\epsilon_{1}=1-\omega_{p}^{2} / \omega^{2}<0$ (see also [13, 14] ).

We show in Fig. 2 the curves associated with the dispersion relations of SPPs propagating along a gold/air and gold/glass interface respectively. Fig. 2A represents the dependencies $\omega$ versus $k_{x}^{\prime}$ corresponding to Eq. 3 . Fig. $2 \mathrm{~B}$ shows the dependencies $\omega$ versus $L_{S P P}$ where $L_{S P P}=1 /\left(2 k_{x}^{\prime \prime}\right)$ is the propagation length of the SPP waves (for the metal optical constant we used the experimental values given in [15]). The typical back bending around $\lambda=$ $520 \mathrm{~nm}$ corresponds to the resonance associated with the bound electrons and the interband transition (for a good discussion see [17]). Far away from the interband the real part of 
the dispersion is close to the asymptotic light lines: we speak about Zenneck surface modes by opposition to Fano and evanescent modes existing close to the interband [13, 16]. An
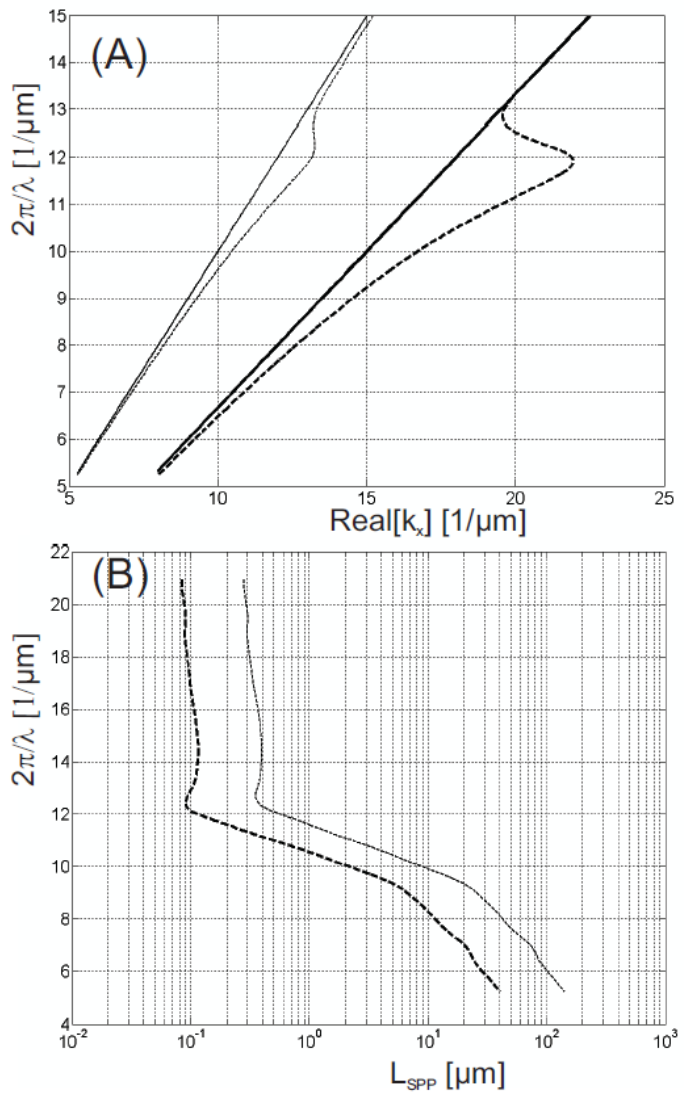

FIG. 2: Dispersion relations of SPP waves propagating along a air/gold (thin dashed curve) and glass/gold (thick dashed curve) interface. (A) Real part of the dispersion relations showing the evolution of $k_{x}^{\prime}$ with $\omega / c=2 \pi / \lambda$. The light cones corresponding to an optical wave propagating in air and glass (i. e., in the bulk medium) are represented by a thin and thick continuous line respectively. The back bending at $\lambda=520 \mathrm{~nm}$ correspond to the interband resonance. (B) Imaginary part of the dispersion relations showing the evolution of $L_{S P P}$ with $\omega / c=2 \pi / \lambda$. The damping is higher for the glass/gold interface.

important feature occurs close to this interband transition since the slope in Fig. $2 \mathrm{~A}$ is diverging. This means that the group velocity defined by $v_{g}=\partial \omega / \partial k_{x}^{\prime}$ is infinite. One can qualitatively deduce that there is however no paradox with causality by remarking that the propagation length is approaching zero at such wavelengths. Actually the SPP life time $\tau_{S P P}$ defined by $v_{g} \cdot \tau_{S P P} \approx L_{S P P}$ is tending as well to zero. This is a clear signature of the absence of significant SPP propagation in the interband spectral region. This fact actually 
precludes faster than light information transfer by SPP waves and is in agreement with relativistic causality.

A second important feature concerning Fig. $2 \mathrm{~A}$ is that the air/gold dispersion curve is located inside of the light cone for glass defined by the equation $\mathbf{K}^{2}=(\omega / c)^{2} \epsilon_{\text {glass }}$ where $\mathbf{K}$ is a real light wave vector and $\epsilon_{\text {glass }}=2.25$. Writing $\mathbf{K}=\left[K_{x}, 0, K_{z}\right]$ the wavevector of a TM (i. e., p polarized) plane wave propagating away from the interfaces into the glass side one see that SPPs propagating at the air/metal interface can radiate into the glass substrate if the condition

$$
K_{x} \approx \pm \operatorname{Real}\left[(\omega / c) \sqrt{\frac{\epsilon_{0} \epsilon_{1}}{\epsilon_{0}+\epsilon_{1}}}\right]
$$

is approximately fulfilled. Here we neglected the role of the imaginary part in Eq. 3. Similarly one can deduce that none of the SPPs propagating at the two interfaces can radiates into the air side.

In order to have a more complete analysis one must actually consider the problem with two coupled interface 0/1 (glass/metal) and 1/2 (metal/air) supporting SPP waves and separated by a small distance $D$. The two interfaces are coupled by evanescent SPP waves tunnelling through the metal slab. Such a mathematical problem can only be treated numerically by resolving an implicit equation. As for the single interface this equation can be defined directly from Maxwell Equations [11]. However it is much easier and convenient for the following to remark with Raether [1] that Eq. 2 and consequently Eq. 3 are obtained by finding the zeros of the numerator in the Fresnel reflectivity coefficient for a TM wave coming from the dielectric side:

$$
R_{0,1}^{p}=\frac{\left(k_{0} / \epsilon_{0}-k_{1} / \epsilon_{1}\right)}{\left(k_{1} / \epsilon_{1}+k_{0} / \epsilon_{0}\right)},
$$

with $k_{j}= \pm \sqrt{\left((\omega / c)^{2} \epsilon_{j}-k_{x}^{2}\right)}$. Actually Raether [1] reasoned with the denominator of the Fresnel coefficient due to different conventions for the signs of the wave vectors $k_{j}$. However it is remarkable that the result is the same at the end of the calculations. Identically one can thus define the Fresnel coefficient for a TM wave reflected by the slab 0-1-2 [1, 12, 17]:

$$
R_{0,1,2}^{p}=\frac{R_{0,1}^{p}+R_{1,2}^{p} e^{2 i k_{1} D}}{1+R_{0,1}^{p} R_{1,2}^{p} e^{2 i k_{1} D}},
$$

and find the zeros of the numerator, i. e., one can solve the implicit equation

$$
R_{0,1}^{p}+R_{1,2}^{p} e^{2 i k_{1} D}=0
$$


in order to define the SPP dispersion relations. From this equation it follows that

$$
\begin{array}{r}
\left(k_{0} / \epsilon_{0}+k_{1} / \epsilon_{1}\right)\left(k_{2} / \epsilon_{2}-k_{1} / \epsilon_{1}\right) e^{i k_{1} D} \\
+\left(k_{1} / \epsilon_{1}-k_{0} / \epsilon_{0}\right)\left(k_{2} / \epsilon_{2}+k_{1} / \epsilon_{1}\right) e^{-i k_{1} D}=0 .
\end{array}
$$

As for the single interface one has an important relation between the real and imaginary parts of the SPP wave vectors in the different medium:

$$
-k_{0}^{\prime} \cdot k_{0}^{\prime \prime}=(\omega / c)^{2} \epsilon_{1}^{\prime \prime} / 2-k_{1}^{\prime} \cdot k_{1}^{\prime \prime}=-k_{2}^{\prime} \cdot k^{\prime \prime}=k_{x}^{\prime} \cdot k_{x}^{\prime \prime} .
$$

Since we are interested only into the solutions which are decaying along the interface we (in agreement with our previous treatment of the single interface) suppose the condition $k_{x}^{\prime} \cdot k_{x}^{\prime \prime} \geq 0$ satisfied. A second important point is that due to the arbitrariness in the sign of $k_{j}$ there are in fact apriori 8 possibilities for writing Eq. 15. However, Eq. 15 is invariant under the transformation $k_{1} \rightarrow-k_{1}$. This means that the number of apriori possibilities for the sign of $k_{j}$ is reduced from 8 to 4 . This multiplicity was studied by Burke et al. [11] however since for the present purpose we are looking for SPP waves leaking from the air/metal interface into the glass substrate we will consider only the possibility

$$
\begin{aligned}
k_{0} & =-\sqrt{\left((\omega / c)^{2} \epsilon_{0}-k_{x}^{2}\right)} \\
k_{1} & =+\sqrt{\left((\omega / c)^{2} \epsilon_{1}-k_{x}^{2}\right)} \\
k_{2} & =-\sqrt{\left((\omega / c)^{2} \epsilon_{2}-k_{x}^{2}\right)} .
\end{aligned}
$$

The sign of $k_{1}$ is however arbitrary as explained above and we choose it here positive by definition. In order to define a SPP wave leaking into the glass substrate one has thus to substitute Eq. 17 into Eq.15 and find numerically (i. e., by a minimization procedure [18, 19]) the zeros of the implicit equation with variable $k_{x}^{\prime}$ and $k_{x}^{\prime \prime}$. This has to be done only in the quarter of the complex plane corresponding to $k_{x}^{\prime}>0, k_{x}^{\prime \prime}>0$. The quarter $k_{x}^{\prime}<0, k_{x}^{\prime \prime}<0$ must be equivalent due to symmetry and corresponds actually to decaying SPP waves propagating in the negative $\mathrm{x}$ direction. The two other quarters of the complex plane correspond to growing SPP waves along the interface and will be rejected on a physical ground (compare [11]).

Figs. $3 \mathrm{~A}$ and $3 \mathrm{~B}$ show numerical calculations of dispersion relations corresponding to a SPP wave leaking through a gold film from the air side to the glass side. The thickness is taken to be $D=70,50,20$, and $10 \mathrm{~nm}$ respectively. For the value $D \geq 70 \mathrm{~nm}$ the 

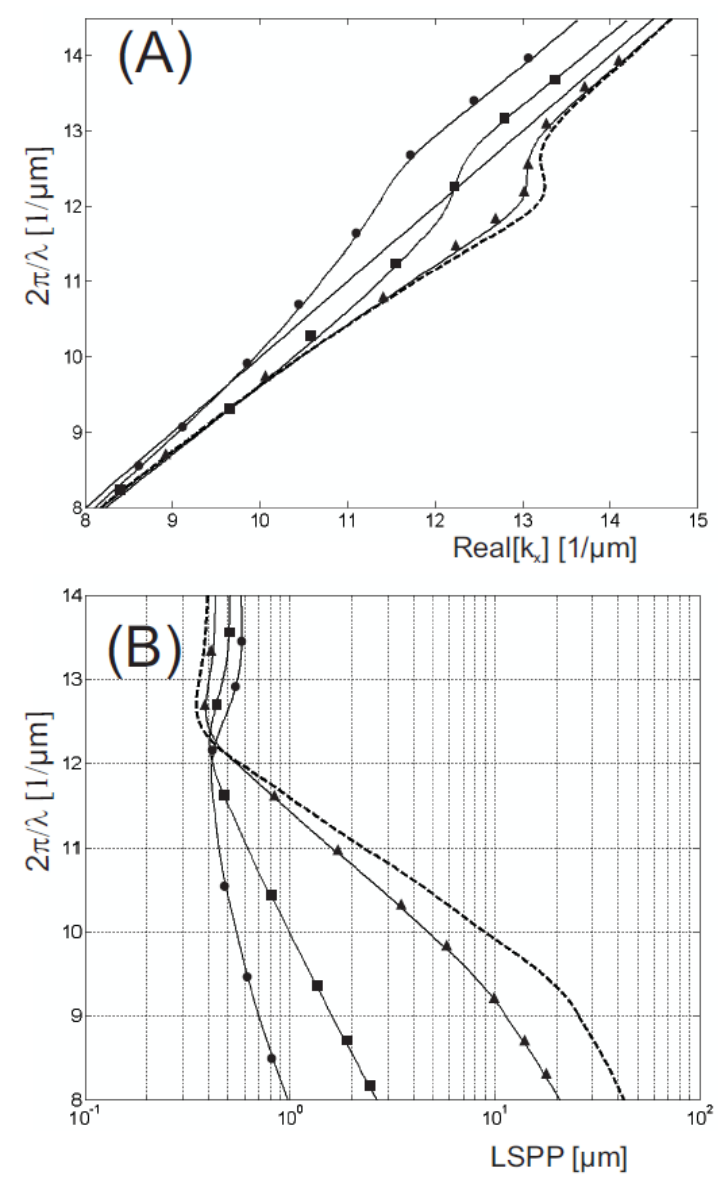

FIG. 3: Dispersion relations of SPP waves propagating along an air/gold interface and leaking into glass. The dispersion is calculated for four film thickness $D: 70 \mathrm{~nm}$ (dashed line curve)es, 50 $\mathrm{nm}$ (continuous line with triangular markers), $20 \mathrm{~nm}$ (continuous line with square markers), and $10 \mathrm{~nm}$ (continuous line with circular markers).(A) Real part of the dispersion relations showing the evolution of $k_{x}^{\prime}$ with $\omega / c=2 \pi / \lambda$. The light cone corresponding to optical wave propagating in air (i. e., in the bulk medium) is represented by a continuous line. (B) Imaginary part of the dispersion relations showing the evolution of $L_{S P P}$ with $\omega / c=2 \pi / \lambda$.

dispersion relation is identical to the dispersion for the single air/gold interface for semi infinite media. However, for smaller thickness the coupling between the interface increases and the propagation length decreases as shown on Fig. 3 B. The magnetic field associated 


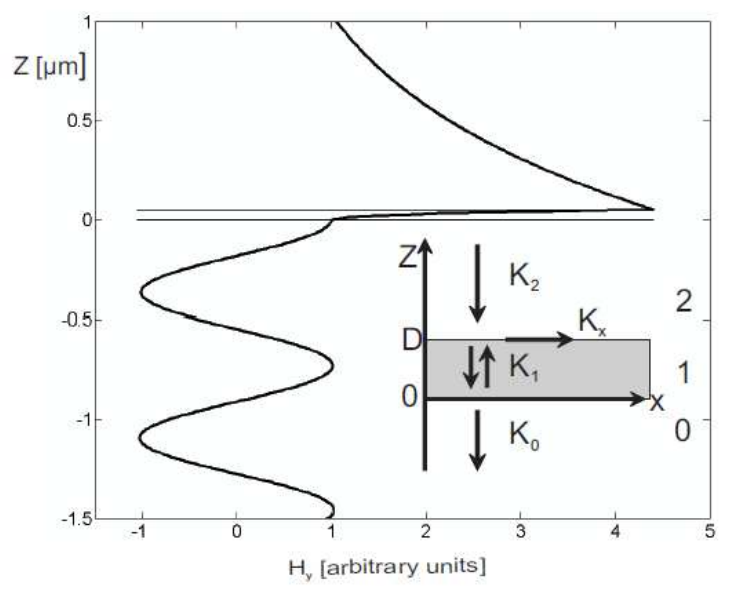

FIG. 4: Structure of the magnetic field Real $\left[H_{y}\right]$ associated with a SPP mode leaking through a gold film of thickness $50 \mathrm{~nm}$ (medium 1) from the air side (medium 2) to the glass side (medium $0)$. The evolution is represented along the normal $z$ to the interfaces. The optical wavelength considered is $\lambda=800 \mathrm{~nm}$. The two horizontal lines show the interfaces separated by $50 \mathrm{~nm}$. The inset shows the conventions for the axis $\mathrm{x}$ and $\mathrm{z}$. The arrows indicate the direction of the real part of the wave vector normally and parallely to the interface.

with SPP electromagnetic modes in this layered system is given by

$$
\begin{array}{r}
H_{0}=e^{i k_{x} x} e^{i k_{0} z} e^{-i \omega t} \\
H_{1}=e^{i k_{x} x}\left[\alpha \sin \left(k_{1} z\right)+\beta \cos \left(k_{1} z\right)\right] e^{-i \omega t} \\
H_{2}=\gamma e^{i k_{x} x} e^{i k_{2}(z-D)} e^{-i \omega t} .
\end{array}
$$

The coefficients $\alpha, \beta, \gamma$ are obtained by considering the boundary conditions and one finds

$$
\begin{array}{r}
\alpha=i \frac{k_{0} \epsilon_{1}}{k_{1} \epsilon_{0}}, \beta=1 \\
\gamma=i \frac{k_{0} \epsilon_{1}}{k_{1} \epsilon_{0}} \sin \left(k_{1} D\right)+\cos \left(k_{1} D\right) .
\end{array}
$$

As an illustration we show in Fig. 4 the evolution of the real part of the magnetic field across a $50 \mathrm{~nm}$ thick gold slab sandwiched between the glass substratum and the air superstratum for an optical wavelength $\lambda=800 \mathrm{~nm}$. As visible the SPP field is located in the vicinity of the air/gold interface and is evanescent on the air side. This is clearly reminiscent of our former analysis of SPPs propagating at the single air/metal. In addition however the wave is leaking radiatively (i. e., propagatively) into the glass substrate. However from Eq. 18 
and the condition $k_{0}^{\prime} \cdot k_{0}^{\prime \prime} \leq 0$ it is clear that the leaking wave is exponentially growing in the $-\mathrm{z}$ direction when going away from the gold slab. This is already the result we obtained when we considered the limit of the thick slab. An exponentially growing wave looks non physical and is in particular associated with infinite radiated energy in the far field. There is now the question of how to connect a growing wave with the basic reasoning giving rise to Eq. 11 and the idea of phase matching between the (real part) of the SPP wave vector with a propagative plane wave vector in the glass substrate. However such paradoxes disappear for two reasons: First, an infinite energy occurs only because we considered an infinite interface or equivalently because we did not consider how the SPP is locally launched on the metal film. When such conditions are taken into account this paradox must disappear [11]. Second, the SPP wave defined by Eq. 18 is actually a wave packet when looked at through the Fourier basis of propagative TM plane waves. Since in the far field (i. e., in the glass substrate) one actually detect such plane waves one must do a Fourier transform in order to generalize Eq. 11 [1, 11]. Instead of Eq. 11 one obtains consequently a statistical distribution of (real) wavevectors $K_{x}$ given by

$$
I\left(K_{x}\right)=\frac{\text { const. }}{\left(K_{x}-k_{x}^{\prime}\right)^{2}+\left(k_{x}^{\prime \prime}\right)^{2}}
$$

where $2 k_{x}^{\prime \prime}$ defines the full width at half maximum (FWHM) of this Lorentz distribution of radiated plane waves. By noting as usual $\theta$ the angle between the wave vectors $\mathbf{K}$ of the plane waves refracted into the glass substrate and the normal $z$ to the interfaces one has by definition $K_{x}=2 \pi \sqrt{\epsilon_{0}} / \lambda \sin \theta$ and the angular distribution of radiated power is in the far field given by:

$$
I(\theta)=\frac{\text { const. }}{\left(2 \pi \sqrt{\epsilon_{0}} / \lambda \sin \theta-k_{x}^{\prime}\right)^{2}+\left(k_{x}^{\prime \prime}\right)^{2}} .
$$

\section{LEAKAGE RADIATION MICROSCOPY}

Historically the first observations of leakage radiation by SPP propagating on a thin metal film were reported by analyzing scattering by rough metal surfaces of light into SPPs [1, 20]. The possibility of using rough surface to excite SPPs was extensively studied in the past [1] and is based on the fact that the scattering by small defects on a flat film can represent a source of evanescent momentum sufficient for the light waves to match the SPP dispersion

relation. Equivalently the amount of momentum needed can be carried by grating coupling 
[1]. SPP waves are subsequently emitted back into the glass substrate as leakage radiation (see Fig. 5). This light collected on a photographic plate forms a ring-like distribution
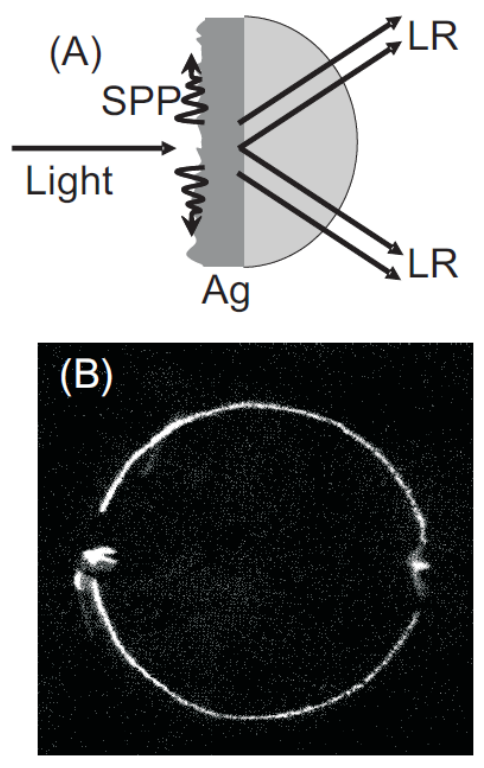

FIG. 5: Observation of leakage radiation (LR) through a rough thin silver film (wavelength $\lambda=$ $550 \mathrm{~nm}$ ). (A) SPPs are launched on the air/silver interface by using scattering by film rugosities to produce the amount of momentum necessary to match the SPP dispersion relation. SPPs are leaking through the glass substrate and detected using a photographic plate in the far-field. (B) Photography showing the leakage radiation cone. The photography is extracted from the work by H. J Simon and J. K. Guha [20] (C) Opt. Comm., Elsevier, 1976).

in agreement with Eqs. 11, 20, 21. The FWHM of the SPP wavevector distribution is in direct correspondence with the radial width of the ring [20]. Further progress was obtained recently with the development of near field scanning optical microscopy (NSOM) which allows the local optical excitation of evanescent waves in the vicinity of a metal surface. Such evanescent waves can carry a sufficient amount of momentum to couple to SPP waves. Direct observations have indeed confirmed this principle [8, 21, 22]. As an example we show in Fig. 6 an experiment in which the NSOM tip launches SPPs on an aluminum film which after interaction with a hole excites optically some quantum dots (QDs) located below. The collected signal shows a specific QD luminescence spectrum [23]. By scanning the sample around the NSOM tip one can realize SPP mapping since the hole acts a probe structure for the field emitted by the tip. Quantitative analysis of the total luninescence of the QDs 
associated with a given hole show clearly that the QD excitation is mediated by SPPS propagating on the aluminum film. Fig. 6B shows the radial dependence of the collected
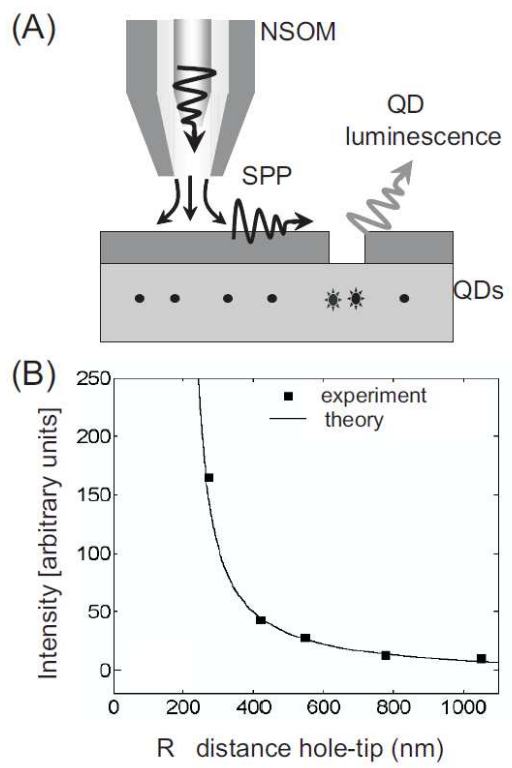

FIG. 6: (A) Sketch of the experiment using a NSOM tip to excite SPPs on an aluminum film with nanoholes. The film containing $240 \mathrm{~nm}$ diameter holes covers a CdTe/ZnTe system of QDs whose spectral luminescence induced by SPP excitation is characteristic of a given hole. The laser excitation wavelength is $514.5 \mathrm{~nm}$. (B) Radial mapping of the SPP intensity. The SPP intensity is proportional to the QDs luminescence of a given hole. The curve represents the evolution of the total luminescence collected (using a detector in the optical far field) as a function of the distance $R$ separating the NSOM (source) from the hole (probe). The experiment is made at $4.2 \mathrm{~K}$ [22] (C) Europhys. Lett., EDP, 2003).

intensity. These results agree well with a 2D SPP dipole model supposing an effective dipole located at the tip apex [8, 22] (see also the discussions concerning the Bethe-Bouwkamp [24 26] theory of diffraction by a small aperture in a metal film in [27-31]). Following this model the SPP wave can be theoretically modelled by a scalar wave $\Psi(\rho, \theta)$ given by

$$
\Psi(\rho, \phi)=\text { const. } \frac{e^{i k_{\mathrm{SPP}} \rho} \cos \phi}{\sqrt{\rho}},
$$

where $k_{\mathrm{SPP}}=k_{x}^{\prime}+i k_{x}^{\prime \prime}$ is given by Eq. $3, \rho, \phi$ are polar coordinates on the metal film and the origin of the coordinate is taken at the dipole position. $\phi$ is the angle between the dipole associated with the NSOM tip (parallel to the polarization of the laser beam injected in the 
NSOM tip) and the the radial vector $\rho=[x, y]$. This simplified model can be theoretically justified by using the Green Dyadic Formalism [32] and has been applied by many authors successfully [8, 9, 22, 33-36] to SPP waves propagating in various environments.

Several authors applied NSOM methods coupled to LRM [8, 9]. In particular in [8] Hecht et al. realized an optical setup using an immersion oil objective to collect the leakage radiation emitted by the NSOM tip on gold or silver films (see fig. 7). The system shown in Fig. 7 B is a

(A)

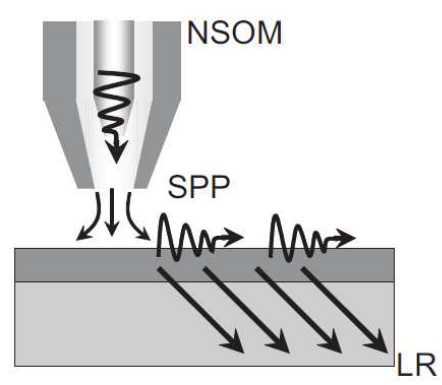

(B)

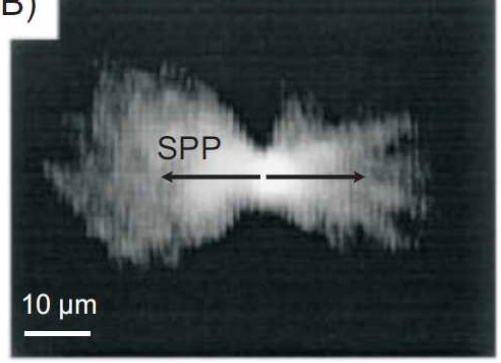

FIG. 7: (A) Principle of the experiment to generate SPP leakage radiation (LR) using a NSOM tip. LR is collected using an immersion oil objective (not shown here). (B) Map of SPP intensity using a charge coupled device (CCD) camera to collect the LR emitted through the glass substrate. SPPs are launched on a $60 \mathrm{~nm}$ thick silver film at the optical laser wavelength $\lambda=633 \mathrm{~nm}$. The image in B is taken from B. Hecht et al. [8] (C) Phys. Rev. Lett, American Physical Society, 1996).

$60 \mathrm{~nm}$ thick silver film optically excited by a NSOM tip at the laser wavelength $\lambda=633 \mathrm{~nm}$. It can be shown by analyzing Fig. $7 \mathrm{~B}$ that the radiation pattern is well described by a 2D dipole model in agreement with Eq. 22. In particular the SPP propagation length was measured and is in fair agreement with our analysis in Sect. 1. Additionally it was shown in [8] that one can also analyze the Fourier distribution of SPP momentum (given by Eqs. 20, 21) by defocusing the objective lens. As expected SPP rings similar to the one of Fig. 5 were observed. 
In the same context we developed in recent years a systematic approach using far-field

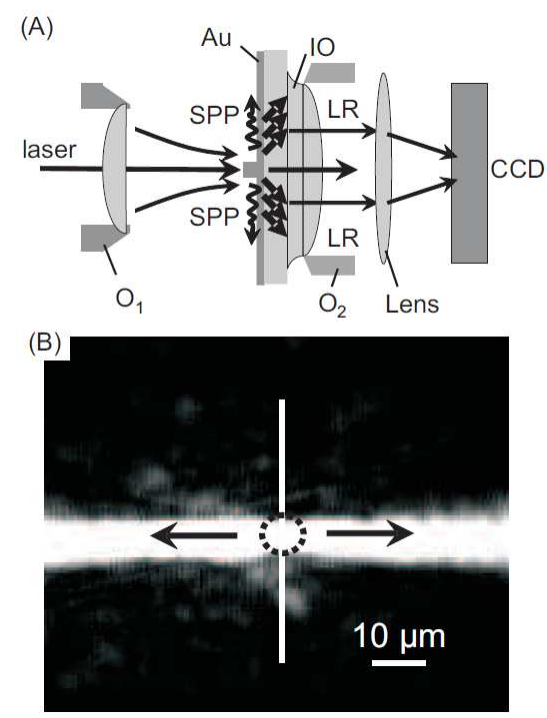

FIG. 8: (A) Principle of the experiment to generate SPP leakage radiation (LR) using a focussed laser beam (objective $O_{1}, 10 \mathrm{x}$, numerical aperture $N A=0.3$ ). LR is collected using an immersion oil objective (objective $\mathrm{O}_{2}, 63 \mathrm{x}, \mathrm{NA}=1.3$ ) and refocussed on the CCD camera by using an auxiliary lens. (B) Map of SPP intensity using a CCD camera to collect the LR emitted through the glass substrate. SPPs are launched on a $50 \mathrm{~nm}$ gold film at the optical laser wavelength $\lambda=800 \mathrm{~nm}$ from a gold ridge (50 nm height, $150 \mathrm{~nm}$ width) represented by a white line. The size of the laser spot with a diameter $\simeq 8 \mu \mathrm{m}$ is represented by the white dashed circle.

microscopy to analyze quantitatively the interaction between SPPs and plasmonic devices by using LRM. The nanodevices studied were all fabricated by electron beam lithography (EBL) allowing the precise and reproducible tailoring of metal and dielectric surfaces on a lateral size dimension down to $20 \mathrm{~nm}$ [37]. As an example we show in Fig. 8 B a LRM image obtained by using a gold ridge (50 nm height, $150 \mathrm{~nm}$ width) lithographed on a $50 \mathrm{~nm}$ thick gold film to launch two well collimated and counter propagating SPP beams. These beams are launched by focussing a laser beam with a microscope objective (10x, numerical aperture $N A=0.3)$ on the gold ridge. Scattering by the nanostructure gives rise to evanescent waves supplying the right amount of momentum necessary for generating a SPP wave. The optical LRM setup is sketched in Fig. 8 A. Leakage radiation emitted through the glass substrate is collected by an immersion oil objective (63x, numerical aperture, $N A=1.3)$. Light is subsequently refocussed on a charge coupled device (CCD) camera. The direct mapping of 
the SPP intensity with this method provides a one-to-one correspondence between the 2D SPP intensity and the image recorded on the CCD camera. It should be observed that an incident laser beam with diameter $D x$ is in the focal plane (object plane) of the objective $O_{1}$ focussed into a disc of diameter $w$ (i. e., $w=$ beam waist) such that

$$
\tan \alpha=\frac{2 \lambda}{\pi w}=\frac{D x}{2 f}
$$

where $f$ is the focal length of the objective and $\alpha$ the divergence angle of the laser beam focussed on the sample. The direct application of this version of the Heisenberg relation [38] implies that the divergence angle $\alpha_{S P P}$ of the SPP beam launched on the metal film must equal the divergence angle $\alpha$ of the impinging laser beam. This result is in good agreement with the experimental case shown in Fig. 8 with $D x=2 \mathrm{~mm}, w=8.3 \mu \mathrm{m}$ and $\alpha=3.5^{\circ}$. Changing the objective focal length is a straightforward means to obtain different divergence angles $\alpha_{S P P}$ (see for example [10, 39, 40]).

As a further improvement it is possible to modify the previous optical setup in order to

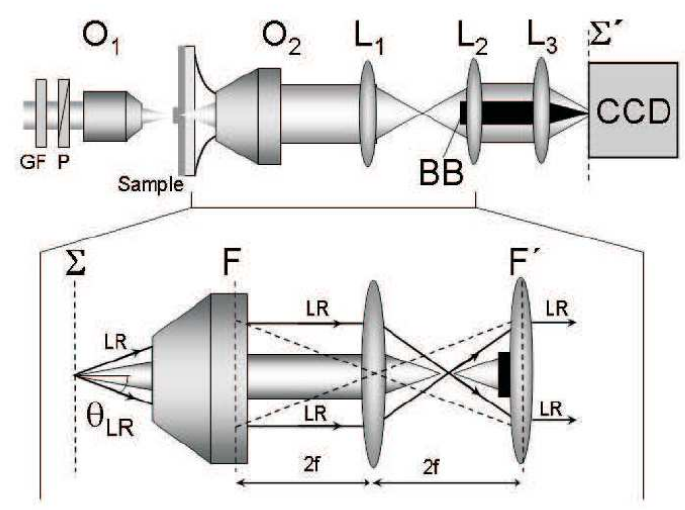

FIG. 9: Experimental scheme for LRM. SPPs are excited by laser light focused by the microscope objective O1 (50x, numerical aperture, $N A=0.7)$ onto a structured gold thin film on a glass substrate. LR emitted into the glass substrate from the gold/glass interface $\Sigma$ is collected by the immersion microscopy objective $\mathrm{O} 2(63 \mathrm{x}, \mathrm{NA}=1.25)$ and imaged by a CCD camera. Depending on the lateral position of the convex lens L3 either the back focal plane or the sample plane is imaged. BB beam block, L1,2,3 convex lenses, $\mathrm{f}$ focal length of L1 and L2; $\Sigma, \Sigma^{\prime}$, sample plane and image thereof; F, F' back focal plane and image thereof [39].

image not only the direct space information but also the momentum corresponding to the 
Fourier space. It is indeed a well known fact of Fourier optics [17, 38, 41] that such a mapping of the wave vector distribution (as given by Eq. 20) is in principle always possible by recording the LR light in the back focal plane $F$ of the oil immersion objective. In the optical setup shown in Fig. 9 [39] we realized a dual microscope able to image SPP propagation in both the direct and Fourier space. In particular the back focal plane $F$ of the oil immersion objective $\mathrm{O}_{2}$ imaged onto a CCD camera in Fig. 9. With such a microscope
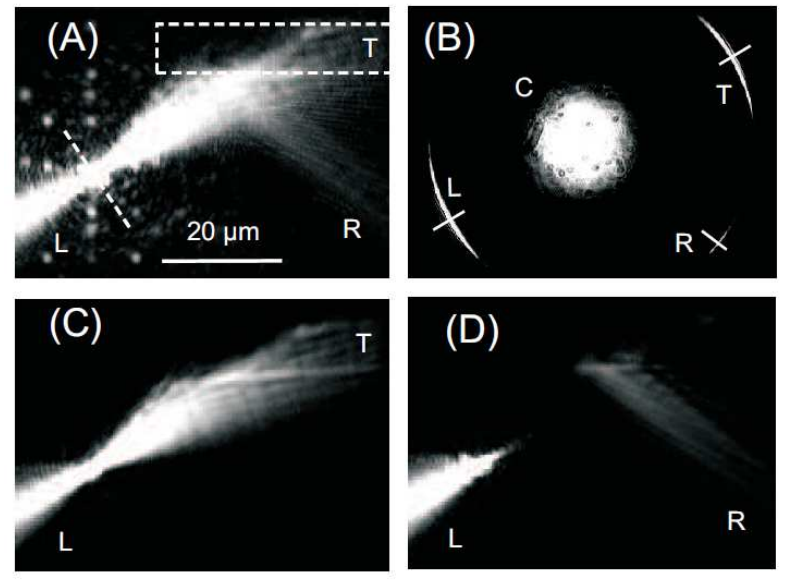

FIG. 10: (A) Direct space LRM imaging of two SPPs beams launched from the ridge and propagating to the lower left $(L)$ and upper right to be partly reflected $(R)$ and transmitted $(T)$ by the Bragg mirror. The image is taken with no beam block. Ridge and Bragg mirror are indicated by the dashed line and rectangle, respectively. (B) Fourier plane LRM imaging corresponding to (A). $\mathrm{C}$ indicates the wave vector components of the directly transmitted laser beam $C ; R, L, T$ have the same meaning as in (A). (C)-(D) are Fourier filtered images after removing the central beam $C$ with a beam block in $F^{\prime}$ (see text). In (C) the reflected beam $\mathrm{R}$ is removed by blocking the arc of the SPP ring $R$ shown in (B). In (D) the transmitted beam $\mathrm{T}$ and the beam incident on the Bragg mirror are removed by blocking the arc of SPP ring $T$ in (B). Data from [39] (C), Appl. Phys. Lett., American Institute of physics, 2006).

it is furthermore possible to act experimentally in the Fourier space image plane $F^{\prime}$. First we can thereby remove the directly transmitted laser beam by using a beam block located on the optical axis. As an application of this method of filtering we consider the reflection of a SPP beam by an in-plane Bragg mirror. SPPs are launched as before from a gold ridge (50 nm height, $150 \mathrm{~nm}$ width) lithographed on a $50 \mathrm{~nm}$ thick gold film. The Bragg mirror [41] 
constitutes a one-dimensional lattice of parallel gold ridges (50 nm height, $140 \mathrm{~nm}$ width) separated by a distance $P$ defining the period of the lattice. The period $P$ is connected to the SPP wavelength by $\lambda_{S P P}=2 \pi / k_{S P P}<\lambda$ and to the angle of incidence reflection $\theta_{S P P}$ of the SPP beam relatively to the (in plane) normal to the lattice by

$$
P=\frac{\lambda_{S P P}}{2 \cos \theta_{S P P}} \text {. }
$$

In the present case shown in Fig. 10 the Bragg mirror is optimized for $\lambda=800 \mathrm{~nm}$ (i. e., $\left.\lambda_{S P P}=785 \mathrm{~nm}\right)$ and for $\theta_{S P P}=45^{\circ}$ incidence angle which means $P \simeq 555 \mathrm{~nm}$. The experimental analysis of such a Bragg mirror when the resonance condition $\left(\lambda, \theta_{S P P}\right)$ is fulfilled reveals a very high reflectivity of $R \simeq 95 \%$ (see, for example, [34] for some earlier results on SPP Bragg mirrors studied with fluorescence microscopy). However, in the present experiment we choose an incident angle $\theta=65^{\circ}$. As a consequence the reflectivity was much lower (see Fig. $10 \mathrm{~A}$ and more details in [39]). This configuration
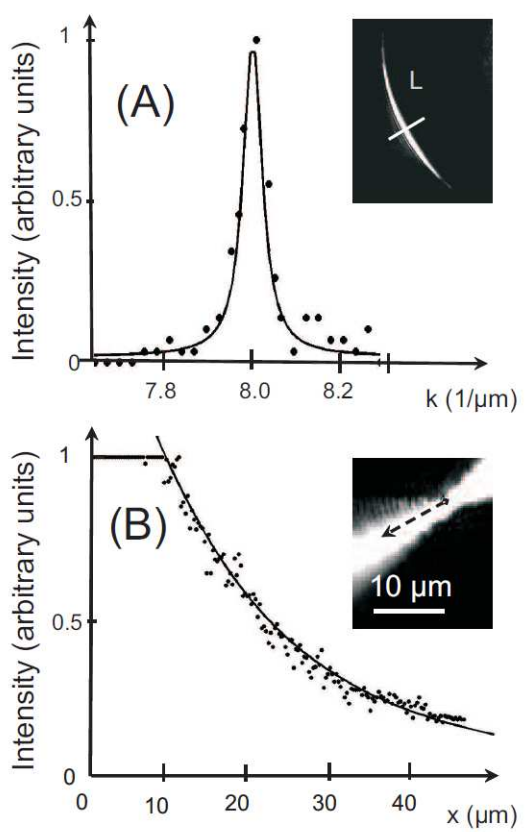

FIG. 11: (A) Fourier space cross-cut along the short solid line in Fig. 10 B corresponding to the $L$ beam (see inset). Experimental data (symbols) are compared with a Lorentz fit (solid curve). The intensity is normalized by setting the maximum intensity of $L$ to 1 . (B) Direct space cross-cut along the L beam in Fig. 10 A. Data points (symbols) are compared to an exponential fit (solid line). Data from [39] (C), Appl. phys. Lett., American Institute of physics, 2006).

reveals SPP interferences in the vicinity of the mirror (Fig. 10 A). In Fig. 10 B we show the 
corresponding Fourier space image. The different observed arcs of LR rings correspond to the reflected $(\mathrm{R})$, and transmitted plus incident $(\mathrm{T})$ beams. The $\mathrm{L}$ beam is associated with a SPP launched in the direction to the left, i. e, away from the mirror. C is the directly transmitted laser beam distribution. By acting in the Fourier plane image $F^{\prime}$ of the LRM microscope we now block the information associated with the central beam and with the $\mathrm{R}$ or T beams [39]. Thereby the according SPP beam images are erased from the image plane and consequently weak intensity beams otherwise observed by interference can be directly analyzed. For further analysis we extracted radial cross-cuts along the white lines as shown in Fig. 10 B [39]. Results are shown in Fig. 11 A for the cross cut along L. The data points agree very well with a Lorentz fit given by Eqs. 20, 21. The FWHM of the Lorentzian distribution gives us a SPP propagation length of $L_{S P P}=20 \mu \mathrm{m}$. This value is in perfect agreement with the cross-cut made along the beam $L$ in the direct space image 10 A (see Fig. 11 B). Both data agree also with values given by the dispersion relations discussed in sect. 1 (see Figs. 2B and 3B).

LRM was subsequently applied by us to many SPP in-plane devices such as beam splitters [10], dielectric lenses, prisms [18] and wave-guides [42]. In particular, LRM experiments were compared to near field optical experiments ( photon scanning tunnelling microscopy) and showed good agreement in the cases considered [18, 42]. LRM appears thus in this context as a complementary far-field optical method to NFO such as NSOM. LRM was applied as well for analyzing SPP Bragg mirrors (with high reflectivity $R \simeq 90-95 \%$ ), interferometers [40, 43] and SPP elliptical cavities [35] or 2D SPP microscopes [44]. In this context we observed [40] stationary SPP waves with very high visibility $V=\left(I_{\max }-I_{\min }\right) /\left(I_{\max }+I_{\min }\right)$ by using LRM. This proves directly that SPP wave coherence is conserved in LRM and can exploited for quantitative analysis down to the spatial resolution limit $\lambda_{S P P} / 2$. Actually SPP interferometers such as the ones described in [34, 35, 43] reveal clear interference pattern and oscillation characteristics of these devices. It is thus possible to develop 2D interferometry for SPP waves having all the advantages of current macroscopic interferometry techniques.

We also mention the realization of plasmonic crystals (i. e., photonic crystals for SPPs) which were studied using LRM (see Fig. 12). In such devices [45] rectangular 2D latices made of gold nano-protrusions (200 nm diameter, $50 \mathrm{~nm}$ height) deposited on a $50 \mathrm{~nm}$ thick gold film (see Fig. 12B) are used to create photonic band gaps at specific SPP 


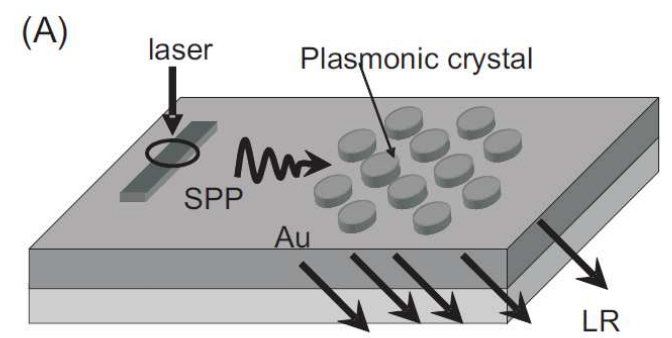

(B)

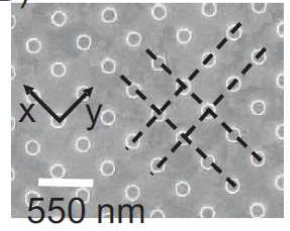

(D)

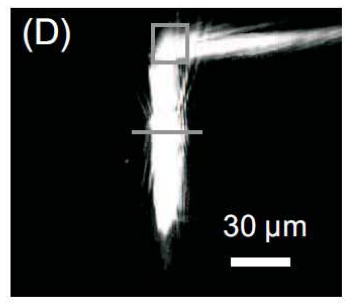

(C)

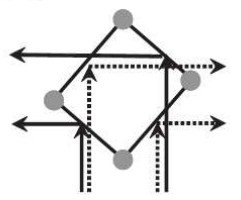

FIG. 12: (A) Principle of SPP in-plane wavelength demultiplexing using plasmonic crystal. SPPs launched from a gold ridge (50 $\mathrm{nm}$ height, $150 \mathrm{~nm}$ width) are propagating in the direction of a 2D rectangular lattice (plasmonic crystal) as described in the text. (B) SEM images of a part of the crystal, Bragg lines are indicated by dashed lines. Such crystals can be seen as two sets of Bragg mirrors perpendiculary oriented to each other. (C) Sketch representing the unit rectangular cell and the direction of the reflected SPP beam for the two different wavelengths at which the Bragg reflections occur. (D, E) LRM images of SPP interacting with the demultiplexer at SPP wavelengths $\lambda_{x} \simeq 730 \mathrm{~nm}$ and $\lambda_{y} \simeq 784 \mathrm{~nm}$, respectively. The gold ridge and plasmonic crystal are indicated by the gray line and the gray box , respectively. The vector basis $\mathbf{x}, \mathbf{y}$ of the latices is indicated in A and E. Data from [45] (C) Nanoletters, American Chemical Society, 2007).

wavelengths $\lambda_{x} \simeq 730 \mathrm{~nm}$ and $\lambda_{y} \simeq 784 \mathrm{~nm}$ (i. e., laser wavelengths of respectively $750 \mathrm{~nm}$ and $800 \mathrm{~nm}$ ) corresponding to the two periods of the lattice $P_{x}=\lambda_{x} / \sqrt{2}=516 \mathrm{~nm}$ and $P_{y}=\lambda_{y} / \sqrt{2}=554 \mathrm{~nm}$. The existence of these band gaps implies that SPP plane waves impinging on small devices build up with such lattice will generate stationary waves in the crystal. More precisely this implies that SPPs will be reflected in specific and different directions if their wavelengths match the values $\lambda_{x}$ or $\lambda_{y}$ and if the angle of incidence relatively to the normal to the Bragg planes of the crystal (Figs. $12 \mathrm{~B}, \mathrm{C}$ ) equals $45^{\circ}$. 
Such devices act consequently as an efficient in-plane wavelength demultiplexer for SPPs [45] as it was indeed observed experimentally (see Fig. 12D and E). While the results discussed here were achieved within the visible spectral range, plasmonic crystal devices are expected to perform even better (e.g., in terms of spectral selectivity) in the infrared (telecom) spectral range due to significantly lower ohmic losses [46]. In general, the use

of multiplexers, splitters and tritters [45] in photonic applications might be specifically appealing due to their small footprint in the range of $10 \times 10 \mu \mathrm{m}^{2}$. Furthermore, the use as building blocks for classical [38] or quantum [47] optical computing can be envisaged.

\section{CONCLUSION}

In this article we reviewed the field of leakage radiation microscopy (LRM) theoretically and experimentally. Theoretically we analyzed how SPP can generate leaky wave in the glass substrate by tunnelling from the air side through a thin metal film supporting SPP waves. We showed in particular that for thick film ( $D \geq 70 \mathrm{~nm}$ ) leakage radiation (LR) does not affect the dispersion relation on the air/metal interface. Importantly the angular distribution of LR is located on a cone matching the SPP dispersion relation. We also reviewed the first experimental results reporting the observation of LR on rough surface and using near field optics methods. We analyzed more recent application of LRM to SPP nanodevices fabricated by electron beam lithography. From all these results we can conclude that LRM is a convenient and versatile far field optical method for analyzing quantitatively SPP propagation on flat film and their interaction with various nano-devices of direct practical interest. Such versatility positions LRM as an appealing alternative to near field optics for studying SPP propagation on a scale of, or larger than the wavelength.

For financial support the European Union, under project FP6 2002-IST-1-507879 is acknowledged.

[1] H. Raether, Surface Plasmons(Springer, Berlin, 1988).

[2] W. L. Barnes, A. Dereux, T. W. Ebbesen, Nature 424, (2003) 824.

[3] C. Genet, T. W. Ebbesen, Nature 445, (2007) 39. 
[4] A. Drezet, A. Hohenau, J. R. Krenn, M. Brun, S. Huant, Micron 38, (2007) 427.

[5] D. W. Pohl, W. Denk, M. Lanz, Appl. Phys. Let. 44, (1994) 651.

[6] D. Courjon, Near-field microscopy and near-field optics ( Imperial College Press, London, 2003).

[7] J. R. Krenn, A. Dereux, J. C. Weeber, E. Bourillot, Y. Lacroute, J. P. Goudonnet, G. Schider, W. Gotschy, A. Leitner, F. R. Aussenegg, C. Girard, Phys. Rev. Lett. 82, (1999) 2590.

[8] B. Hecht, H. Bielefeldt, L. Novotny, Y. Inouye, and D. W. Pohl, Phys. Rev. Lett. 77, (1996) 1889.

[9] A. Bouhelier, Th. Huser, H. Tamaru, H. -J. Güntherodt, D. W. Pohl, Fadi I. Baida and D. Van Labeke, Phys. Rev. B. 63, (2001) 155404.

[10] A. Stepanov, J. R. Krenn, H. Ditlbacher, A. Hohenau, A. Drezet, B. Steinberger, A. Leitner, and F. Aussenegg, Opt. Lett. 30,(2005) 1524.

[11] J. J. Burke, G. I. Stegeman, T. Tamir, Phys. Rev. B 33, (1986) 5186.

[12] J. D. Jackson, Classical Electrodynamics (J. Wiley and Sons, New York, 1975).

[13] G. N. ZhiZhin, M. A. Moskalova, E. V. Shomina and V. A. Yakovlev in Surface Polaritons, edited by V. Agranovich and D. L. Mills (North-Holland Publishing Company, Amsterdam, 1982) pp. 93-143.

[14] A. V. Kats, S. Savel'ev, V. A. Yampol'skii, F. Nori, Phys. Rev. Lett. 98, (2007) 073901.

[15] P. B. Johnson and R. W. Christy, Phys. Rev. B 6, (1972) 4370.

[16] P. Halevi in Electromagnetic Surface modes edited by A. E. Boardman (John Wiley, New York, 1982) Chap. 7.

[17] L. Novotny, B. Hecht, Principles of Nano-Optics (Cambridge Press, London 2006).

[18] A. Hohenau, J. R. Krenn, A. L. Stepanov, A. Drezet, H. Ditlbacher, B. Steinberger, A. Leitner, F. R. Ausseneg, Opt. Lett. 30, (2005) 893.

[19] J. A. Dionne, L. A. Sweatlock, H. A. Atwater, A. Polman, Phys. Rev. B 72, (2005) 075405.

[20] H. J Simon, J. K. Guha, Opt. Comm. 18, (1976) 391.

[21] C. Sönnichsen, A. C. Duch, G. Steiniger, M. Koch, G. vonPlessen, J. Feldmann, Appl. Phys. Lett. 76, (2000) 140.

[22] M. Brun, A. Drezet, H. Mariette, N. Chevalier, J. C. Woehl, S. Huant, Europhys. Lett. 64, (2003) 634.

[23] M. Brun, S. Huant, J. C. Woehl, J.-F. Motte, L. Marsal, H. Mariette, Solid State Comm. 121, 
(2002) 407.

[24] H. A. Bethe, Phys. Rev. 66, (1944) 163.

[25] C. J. Bouwkamp, Philips Res. Rep. 5, (1950) 321.

[26] C. J. Bouwkamp, Philips Res. Rep. 5, (1950) 401.

[27] C. Obermuüller and K. Karrai, Appl. Phys. Lett. 67, (1995) 3408.

[28] C. Obermüller, K. Karrai, G. Kolb, and G. Abstreiter, Ultramicroscopy 61, (1995) 171.

[29] A. Drezet, J. C. Woehl, and S. Huant, Europhys. Lett. 54, (2001) 736.

[30] A. Drezet, S. Huant, and J. C. Woehl, Europhys. Lett. 66, (2004) 41.

[31] A. Drezet, J. C. Woehl, and S. Huant, Phys. Rev. E65, (2002) 046611.

[32] T. Søndergaard, S. I. Bozhevolnyi, Phys. Rev. B69, (2004) 045422.

[33] H. Ditlbacher, J. R. Krenn, N. Felidj, B. Lamprecht, G. Schider, M. Salemo, A. Leitner, and F. R. Aussenegg, Appl. Phys. Lett. 80, (2002) 404.

[34] H. Ditlbacher, J. R. Krenn, G. Schider, A. Leitner, and F. R. Aussenegg, Appl. Phys. Lett. 81, (2002) 1762.

[35] A. Drezet, A. L. Stepanov, H. Ditlbacher, A. Hohenau, B. Steinberger, F. R. Aussenegg, A. Leitner, and J. R. Krenn, Appl. Phys. Lett. 86, (2005) 074104.

[36] J.-Y. Laluet, E. Devaux, C. Genet and T. W. Ebbesen, J.-C. Weeber and A. Dereux, Opt. Express 15, (2007) 3488.

[37] M. A. McCord and M. J. Rooks, in Handbook of Microlithography, Micromachining and Microfabrication, Volume 1 edited by P. Rai-Choudhury (SPIE and The Institution of Electrical Engineers, Bellingham, Washington, 1997), pp. 139-249.

[38] Saleh, B. E. A., Teich, M. C. Fundamentals of Photonics (Wiley, New York, 1991).

[39] A. Drezet, A. Hohenau, A. Stepanov, H. Ditlbacher, B. Steinberger, N. Galler, F. R. Aussenegg, A. Leitner, J. R. Krenn, Appl. Phys. Lett. 89, (2006) 091117.

[40] A. Drezet, A. L. Stepanov, A. Hohenau, B. Steinberger, N. Galler, H. Ditlbacher, A. Leitner, F. R. Aussenegg, J. R. Krenn, M. U. Gonzalez, J.-C. Weeber, Europhys. Lett. 74, (2006) 693.

[41] M. Born and E. Wolf, Principles of Optics, seventh (expanded) edition (Cambridge University Press, Cambridge, 1999).

[42] B. Steinberger, A. Hohenau, H. Ditlbacher, A. L. Stepanov, A. Drezet, F. R. Aussenegg, A. Leitner, J. R. Krenn Appl. Phys. Lett. 88, (2006) 09410 .

[43] A. Drezet, A. Hohenau, A. Stepanov, H. Ditlbacher, B. Steinberger, F. R. Aussenegg, A. 
Leitner, J. R. Krenn, Plasmonics 1, (2006) 141.

[44] A. Drezet, D. Koller, A. Hohenau, F. R. Aussenegg, A. Leitner, J. R. Krenn , submitted (2007).

[45] A. Drezet, D. Koller, A. Hohenau, J. R. Krenn, Nanoletters (Mai 2007) DOI: 10.1021/nl070682p.

[46] T. Nikolajsen, K. Leosson, I. Salakhutdinov, S. I. Bozhevolnyi Appl. Phys. Lett. 82, (2003) 668.

[47] E. Knill, R. Laflamme, G. J. Milburn, Nature 409, (2001) 46. 